\title{
HOLISTIC 3D DIGITAL DOCUMENTATION OF A BYZANTINE CHURCH
}

\author{
A. Anastasiou, E. Syrokou, S. Tapinaki, A. Georgopoulos \\ Laboratory of Photogrammetry, School of Rural \& Surveying Engineering, National Technical University of Athens, Greece. \\ anastasia1808@gmail.com, syrokoulena@gmail.com,sevi@survey.ntua.gr,drag@central.ntua.gr
}

\section{Commission II, WG II/8}

KEY WORDS: Geometric Documentation, 3D Model, Textured Model, TheoLt, Laser Scanner, Stereoscopic Video, Cultural Heritage

\begin{abstract}
:
The aim of the present paper is the geometric documentation of the church of St Spyridon using modern digital methods of data collection and processing. The church is located in the Medieval City of Rhodes and the residues of several different historical phases found in the church prove the rarity and the amount of alterations it underwent over the years.

Geodetic measurements, laser scanning and acquisition of photographic data were performed, in order to construct the 3D model of the church. 23 drawings were drafted at a scale of 1:50, including horizontal sections, exterior and vertical sections. The projected information of each drawing is described with the help of the corresponding orthophotographs. Moreover, the three-dimensional photorealistic model (textured model) of the church was created, as well as a stereoscopic video and interactive virtual tour, via the 3DHOP platform.
\end{abstract}

\section{INTRODUCTION}

The object of the present study is the Early Christian church of St. Spyridon in the Medieval City of Rhodes (Figure 1). This paper presents all the work carried out using modern digital methods of data collection and processing for its geometric documentation, which is the main objective of the work, in order to produce detailed drawings at a scale of 1:50, as well as the three-dimensional photorealistic digital model of the church, a video of the $3 \mathrm{D}$ model, as well as an interactive tour, using the $3 D H O P$ platform. All the above are set to be utilized for the future restoration of the church by the authority responsible of the monument.

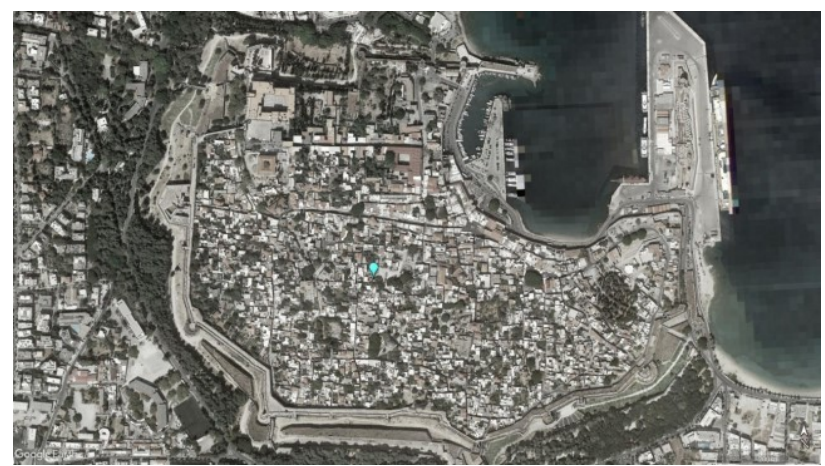

Figure 1. The position of the church in the Medieval City of Rhodes.

The church is located within the walls of the Medieval City of Rhodes in Greece and was firstly built in the 12 th c. AD. Before that, a Hellenistic temple had been constructed at the same spot in the $3^{\text {rd }} \mathrm{c}$. BC. In fact, the foundations of this ancient temple are visible to this day outside the north wall of the church. Since the Ottoman conquest of the island of Rhodes in 1522, all Christian churches were transformed into mosques. As a result, a minaret was added to the church of St. Spyridon and its name subsequently changed to "Kavakli Mescit" (Figure 2).

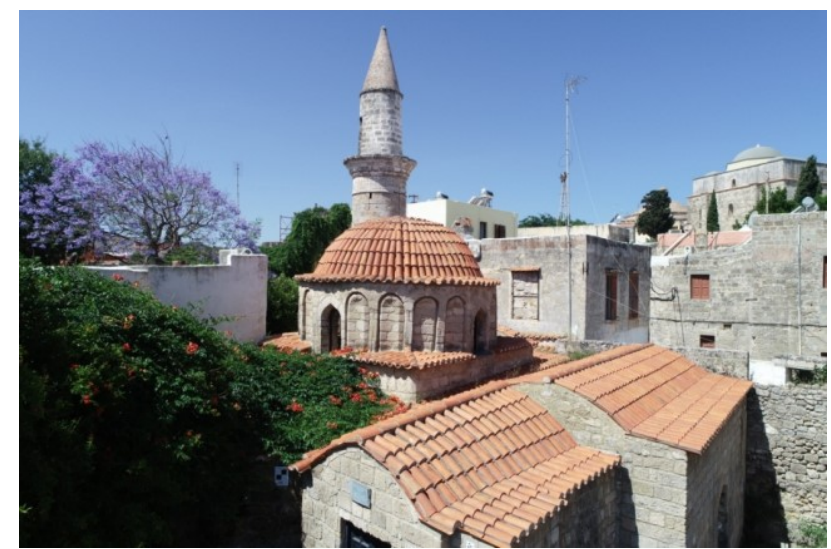

Figure 2. The church of St Spyridon today.

The rarity of the monument and the number of historical phases it went through over the centuries are suggested by the remainders of the construction and decoration. A crypt, dated as far back as the $16^{\text {th }}$ century, containing a mural of the Crucifixion, depicting the sponsors of the church at the time, a father and a son on their knees, is one of its most remarkable features (Figure 3).

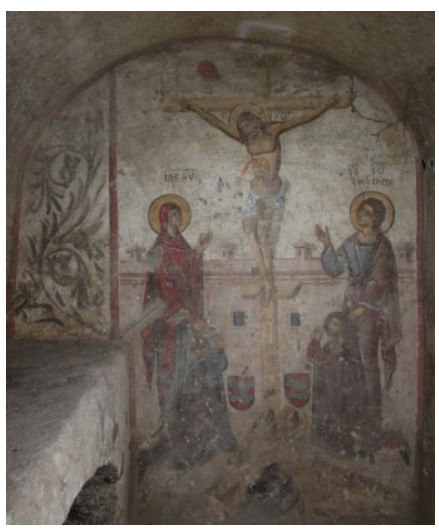

Figure 3. The mural of the Crucifixion in the crypt. 
In 1984, the Ephorate of Byzantine Antiquities of Dodecanese undertook the restoration of the church, while, at the same time, conducting excavations inside and outside the church. These excavations revealed nine box-shaped tombs covered by stone plates, as well as a total of 50 skeletons in makeshift burials. Other interesting findings for the history of the monument, reaching back to the Hellenistic era, include rings, coins, pottery etc.

Regarding the architecture of the church today, it is a domed three-aisled basilica containing a crypt and a minaret at its south west corner. The entrance is now located at the north wall of the church, however it is unclear which entrance is the original one, given that there are two other doors located at the west and south of the church. (Volanakis, 2000)

The equipment used for the documentation included a standard total station, for the acquisition of geodetic data and a laser scanner (Faro Focus X130), for the acquisition of point clouds of the object, both externally and internally. Moreover, three DSLR cameras paired with a variety of lenses and a UAV were used for the detailed photography of the church. Due to the complexity of the object the software TheoLt (www.theolt.com) was used, to facilitate the geodetic data collection.

\section{METHODOLOGY}

\subsection{TheoLt Measurements}

In the case of the geometric documentation of the church of St Spyridon TheoLt was used so that the geodetic process was greatly facilitated. TheoLt is a software that helps record each measurement in an Autocad drawing in real time. In short, by connecting the total station to a laptop via USB and using the TheoLt app as well as Autocad, each measurement is transferred instantly in a drawing. (Blake, Grundon, 2008).

In detail, firstly, it is possible to set a local coordinate system by setting the coordinates of the instrument location, as well as the orientation of the coordinate system, by rotating the instrument towards the preferred North direction. Then, reference points can be measured, which will later be used to create a traverse. Moreover, the software calculates the accuracy of each station point, which can be viewed in the tab "Position Verification".

Furthermore, TheoLt can be used for the process of "resection". Resection uses three or more known target survey points to automatically determine the $\mathrm{X}, \mathrm{Y}, \mathrm{Z}$ coordinates of the instrument position. This is a useful process when there is need to place the instrument in a "blind" station, which is not visible from other traverse stations.

Finally, points for 3D polylines can be easily measured and directly drawn, which can be used for the measurement of sections through the object. (Hadjidemetriou, 2015)

Consequently, in this particular application of the software, a traverse of five stations was created commencing on the outside of the church and continuing towards the inside. In addition, five more stations were created inside the church with the method of resection, so that the targets of the laser scanner and the photogrammetric procedures were measured. Finally, after careful observation of the layout of the church, as well as the areas of special interest, seven sections were measured in the surroundings of the church: a horizontal section at $1.3 \mathrm{~m}$ from the floor of the entrance, passing through most of the doors and windows of the church, three transverse sections (east, central and west), three longitudinal sections (south, central and north) and three more in the underground crypt: a horizontal section at $2.5 \mathrm{~m}$ from the floor of the entrance of the church, a transverse and a longitudinal section (Figure 4).

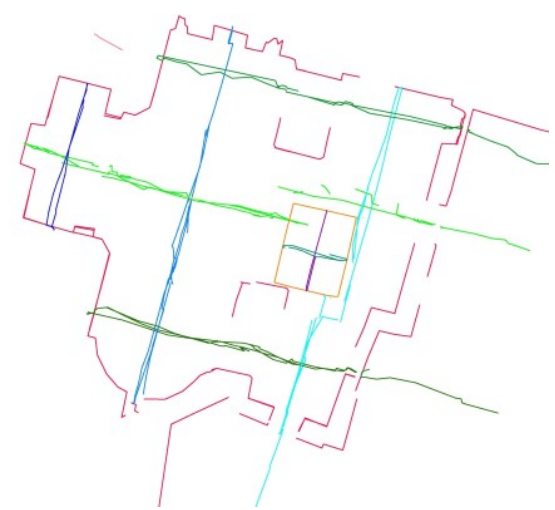

Figure 3. Measured sections using TheoLt.

\subsection{Laser Scanning}

The purpose of the use of laser scanner is the creation of a three-dimensional model of the surface of the object, accurately georeferenced and scaled.

In the case of laser scanner (as well as the case of photography, which will be described in the next chapter), specific targets are required to be placed evenly on the walls of church, both inside and outside. These targets are automatically recognized by the cloud processing software, FARO Scene.

For this purpose, seven targets were placed inside the church, so that at least three of them were visible from each station of the laser scanner and seven more were placed on the external walls of the church. As for the placement of the laser scanner for each scan, the position is selected in the basis of sufficient overlap among the point clouds, so that no gaps are created, and the registration and orientation is possible. Moreover, the option of the density of the gathered points at a specific distance is available, which, in this instance, is set to $1 \mathrm{~cm}$ for a distance of $10 \mathrm{~m}$ from the laser scanner. Finally, 24 scans were performed, with 13 of them being inside the church and the rest outside, with an approximate duration of 20 minutes per scan. Overall, the scans covering the exterior of the church contained more than 736 million points, whereas the scans of the interior contained circa 551 million points.

As for the editing process of the point clouds acquired by the laser scanner, the first step includes their merging and georeferencing, using the FARO Scene software. The purpose of the editing was to modify and reduce the size of the scans, in order to make them more manageable. Some of the specified parameters include a distance filter, which removes points exceeding a user-defined distance, the edge detection filter, which maintains the points that best describe the edges of the building and the auto-detection of the targets. The option of the automatic georeferencing of the scans was not selected, as it was to be performed manually thereafter, using the coordinates of the targets, which derived from the TheoLt measurements.

Finally, the external and internal scans were merged, with a mean error of $\pm 5 \mathrm{~mm}$ and a minimum overlap of $3 \%$ between them. 


\subsection{Image-based Modelling}

Given the complexity of the layout of the church of St Spyridon, the difficulty of access and the lack of visibility in parts of the church, it was decided that ground and aerial photography using a UAV would be carried out.

As a first step, as in the case of the laser scanner, special targets were placed both inside and outside the church to be automatically recognized by Agisoft's Metashape software, which would be then used for the image orientation. The targets were placed taking into account that each target should appear in at least three photographs and in order to achieve the best possible and most uniform coverage of the surfaces of the church.

All the exterior surfaces of the church were photographed using a Canon Mark III full frame DSLR camera and a $24 \mathrm{~mm}$ lens at the late hours of the afternoon so that lighting would be uniform. The photography inside the church was carried out with the use of a Canon 6D full frame DSLR camera and a 24 mm lens, as well as a Canon 80D crop APS-C DSLR camera with an $18 \mathrm{~mm}$ lens. Since there was little natural light through the windows of the dome and the entrance of the church, studio flashes were used for eliminating shadows and in order to achieve uniformity and a natural lighting effect.

As the photography of the entire roof and the minaret was not possible by ground methods, the use of UAV photography was required. For this purpose, the DJI Phantom 4 Pro was used and two flights were scheduled, with one of them focusing on the courtyard and the roof of the church and the second one focusing on the minaret. Overall, the number of pictures taken amounted to 1122 internally (Table 1) and 854 externally (Table 2).

Table 1. Number of pictures taken internally

\begin{tabular}{c|c}
\multicolumn{2}{c}{ internally } \\
\hline Interior & Number of pictures \\
\hline North transept & 498 \\
Nave \& altar & 329 \\
South transept & 152 \\
Crypt & 143 \\
\hline
\end{tabular}

Table 2. Number of pictures taken externally

\begin{tabular}{c|c}
\multicolumn{2}{c}{ externally } \\
\hline Exterior & Number of pictures \\
\hline North wall & 170 \\
Dome & 211 \\
Minaret & 373 \\
Roof & 100 \\
\hline
\end{tabular}

The first stage of the editing process of images includes their orientation, the auto-calibration of the camera and the creation of a sparse point cloud, after finding the relative location of each image, using Agisoft software, Metashape Professional. Subsequently, the sparse point cloud was georeferenced, using the coordinates of the already measured targets and then, a dense point cloud was created.

The automation of this software is related to the Structure from Motion (SfM) algorithm. The purpose of the SfM algorithm is to reconstruct the geometry of the scene and camera movement while capturing the images, using two or more images of the object. The SfM algorithm analyzes two-dimensional (2D) features and actually, detects specific features of the object, such as angles and lines and is able to match them between images. (Westoby et al., 2012) In addition, it defines threedimensional (3D) information deriving from the estimation of the camera movement in relation to the structure of the object. It corrects the bundle of rays and reconstructs the surfaces by triangulating the images and adding a "stereoscopic" perception.

For this task, the images mentioned above were separated into groups, depending on the spaces of the church they depicted. For each set of images, the editing procedures were common. Initially, masks hiding unwanted objects, shadows and non focused areas were manually added to each image. These masks were not active during the creation of the sparse and dense point clouds but were necessary for the later stage of the production of the texture of the model, as well as the production of the orthophotographs.

Subsequently, the targets placed throughout the church were automatically identified by the software and even those not identified were manually set and the coordinates of all the targets were added to the software. Finally, the dense point cloud was created, which was then used to fill the gaps of the laser scanner point cloud.

As for the images obtained with the use of the UAV, their orientation was achieved using the reference files accompanying each image which contained information on the latitude, longitude and altitude. The difference in orientation is that the capture point for each image was predetermined and was not detected by the software.

\subsection{Geometric Documentation}

After the automated creation of the dense point cloud of the church using the MVS procedure, editing was necessary, as detailed above, in the case of the laser scanner point clouds, in order to drastically reduce the volume of the points, as well as their noise. That was achieved with the use of Geomagic Studio software and a number of special algorithms provided by it.

The first algorithm, Select outliers, selects points that do not appear to follow the surface of the object, depending on the degree of sensitivity set by the user. The second algorithm, Reduce noise, does not remove points, but moves the existing ones to describe the surface of the object more accurately. The third and final algorithm used was Uniform, which divides the object into groups of points and maintains the one point that best describes the object from each group. Unlike the first algorithm, it does not allow the user to control which points are to be deleted. It should be noted that all algorithms were implemented more than once, since the volume of the point cloud was still too large to manage. In this way, the editing process was more gradual and controllable.

The next step of the editing process was the creation of the three-dimensional surface using the Triangulated Irregular Network method, still in the environment of Geomagic Studio. The command that was used for the creation of the threedimensional surface was Wrap, after the specification of certain parameters, such as a reduction of the noise of the point clouds anew and the automatic filling of small surface discontinuities. It is worth noting that the large amount of scanning data that was collected, due to the high density of points, is justified by the fact that a complete and detailed description of the shape of the object had to be ensured. The decision for the acquisition of a large amount of data was made on the grounds of difficulty to revisit the church for additional measurements. 
In many cases, the point cloud of the laser scanner appeared incomplete, as the model presented large surface discontinuities and lack of information that could not be manually completed. This resulted in the use of the dense point cloud created by the DSLR and UAV images for the completion of the surface of the model.

However, most of the instances of small discontinuities and imperfections of the surface were handled differently, using the tools provide by Geomagic Studio. The tool that was primarily used was Fill holes, with the option to adjust the curvature of the new surface each time, according to the model requirements. In some cases, inverted triangles or incorrectly inclined triangles had to be deleted and redesigned using the same tool. These incorrect triangles may be due to residual noise of the point clouds. Another tool which facilitated the process of editing the three-dimensional surface was Mesh doctor, which automatically identifies and fixes the cases of overlapping triangles creating sharp edges in places where the surface was supposed to be smoothed, as well as small triangles located off the surface of the church.

The final products of the geometric documentation include the drawings of the facades as well as the horizontal and vertical sections of the church of St Spyridon. These drawings are to be utilized by other experts (archaeologists, architects, civil engineers etc.) who will contribute in the studies for the restoration of the church. For this purpose, each drawing must be realistic, correctly representing the shapes and surfaces of the church and depicting all necessary details.

As mentioned above, after the careful examination of the shape and areas of interest of the church, it was decided that ten sections would be measured, using the TheoLt software. Another criterion for sections was for them to be parallel to the object, in order to avoid distortions due to projection. Also, all vertical transverse sections are parallel to each other and the same applies to all vertical longitudinal sections. In some cases, e.g. the central transverse section passing through the center of the dome, a partial parallel displacement was decided, so that the section would also pass through the entrance of the church.

Section points were determined with the help of Geomagic software. For the definition and creation of the plane of each section, three points were necessary each time, using Geomagic Qualify. Using the same plane as well as the three-dimensional surface of the object turned back into points, the line for each section was created, each time defining the width of the plane, depending on the level of detail required. Afterwards, the points making up each section were joined using Autodesk's Autocad, so that the section line emerged.

Finally, using the planes for each section, along with the section lines, the three-dimensional model and the oriented images, orthophotographs were produced, using Agisoft's Metashape. Each orthophotograph was then carefully examined in respect to the projected elements, as well as its radiometry. Some of the problems often encountered included blurry areas, highly bright or highly shaded areas, incorrectly displayed objects, as well as areas with no color information at all. All the above were corrected using the tools provided by Metashape, especially with the manual selection of the images from which the final orthophotographs would be produced.

The final drawings were produced in the Autodesk AutoCAD environment. Each drawing consists of the corresponding section line and the orthophotographs describing the projected objects. As stated above, the areas lacking information were marked with hatched polygons and their outline was manually drawn, with the roof tiles being a typical example. In addition, in order to create a sense of depth in each drawing, the brightness of the orthophotographs varied, so the furthest areas would be visualized as brighter. Finally, essential elements for a complete and legible drawing were added, such as a geodetic canvas, an elevation marker, a graphic scale, a memorandum and information plate indicating the number of the drawing, the scale and the authors, as well as a key-drawing, indicating the trace of the section depicted $\mathrm{n}$ the drawing (Figure 5).

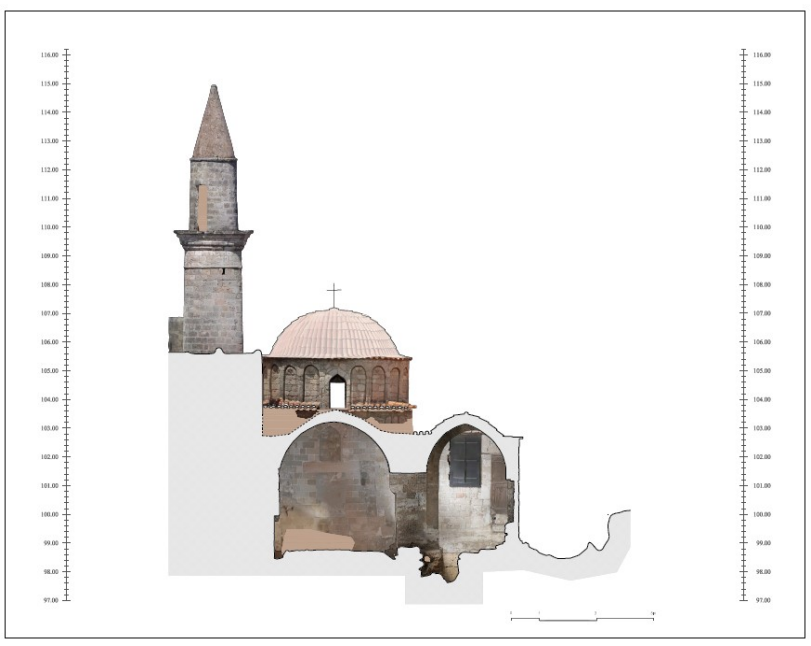

Figure 4. Drawing of a vertical transverse section.

Overall, 23 drawings were produced, including horizontal and vertical sections describing both the interior and the exterior of the church, in addition to the three accessible façades and the top layout of the church. A number of control inspections were necessary among the drawings, to ensure the horizontal and elevation errors were within acceptable limits. Initially, the vertical sections were compared with the horizontal section. Another check included the comparison among all vertical sections. Essentially, the inspection concerned the correspondence of geometric information in characteristic parts of the church, such as the columns, the windows etc. among vertical transverse and longitudinal sections. Moreover, inspections were required for the dome of the church, which was, at first, believed to be hemispherical. After certain tests using circles at different levels, with the help of several horizontal sections made on the dome, the conclusion was that the dome was not hemispherical, possibly due to the lack of experience of the craftsmen who worked on the construction at the time.

It is worth noting that deviations of both the three-dimensional model and the drawings range between $5 \mathrm{~mm}$ and $10 \mathrm{~mm}$, the difference being under the $12.5 \mathrm{~mm}$ limit dictated by the $1: 50$ scale. Therefore, the three-dimensional model which resulted from the processing of the laser scanner and image data corresponds to reality.

\subsection{Texture}

Regarding the three-dimensional model of the church of St Spyridon, the attribution of texture of the model, using Agisoft's Metashape software was the next step. Essentially, a texture map is created for each space of the church, which combines the three-dimensional model with the images, giving it the correct texture. More specifically, an image is created as a development 
of the surface containing the correct colours and texture that emerged with the use of the photographs (Figure 6).
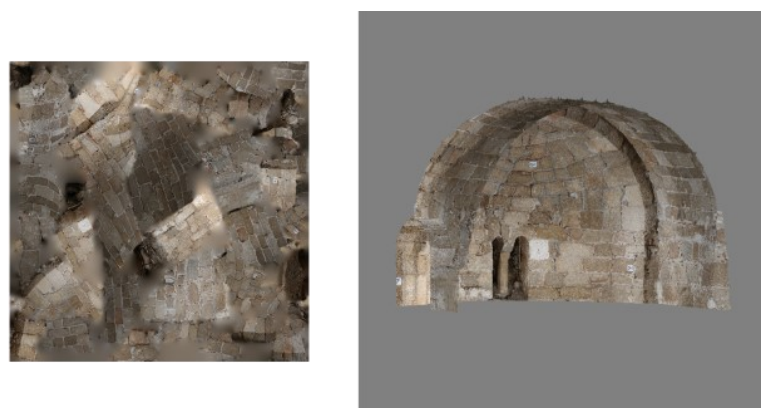

Figure 5. The image of the texture and the textured model of part of the altar.

During this process, the user has the option to set how the surface texture map will be created. Firstly, it is possible to create a predetermined number of texture maps for the entire surface of the model, while secondly, it is preferable to use smaller portions of the surface and create a single texture map for each of these portions. In both cases the resolution of the image is user-defined. In this application, the use of smaller portions of the surface were preferred in order to reduce the time required for the production of each texture map and facilitate data management. The image resolution was set to 4096 x 4096 pixels, which is advantageous and most easily managed resolution by most software. Choosing the right method to create the texture of the model, along with the selection of the most appropriate images for each region helps achieve the best visual quality of the final model.

Initially, each portion of the model is introduced to the software, in continuation of the image orientation. For each space, only the images displaying the corresponding space with sharpness and small radiometric differences stay active, while in many cases additional masks are placed so that only specific portions of the image are used to render the texture. It is important to select as few images as possible, so that the texture map is rapidly created, and the result is as sharp as possible.

Moreover, a series of parameters needs to be specified for the creation of the texture. At first, the Mapping mode parameter specifies how the spread of the texture is going to be condensed into the texture map image. The default option Generic is preferred, as in this case the geometry of the object is not predetermined but is considered arbitrary. Therefore, the software tries to create as uniform a texture as possible. Next, the Blending mode parameter is used to determine how the value of the pixels that will be used for the creation of the texture will be combined. The option Mosaic is used, followed by a two-step procedure. In the first step, the low-frequency areas of the images are combined, using a weighted average pixel value, whose level is derived from a set of parameters, including the distance of said pixel from the center of the image. In the second step, the high-frequency areas, where all the details of the object can be found, are selected from a single image, which presented the corresponding area of interest with high resolution and is in near-orthogonal projection. Basically, only the focused parts in the center of the image are used. In addition, the resolution and number of images where the texture map will be displayed are set to $4096 \mathrm{px} / 1$, as mentioned above (Texture size / count). Finally, the parameters Ghosting filter and Hole filling are activated, for the avoidance of duplicate reflections on the surface and discontinuities on the texture, as well as the creation of noise in complex surfaces which create shows in certain parts of the model, respectively (Agisoft Metashape Development Team, 2019).

This process had to be performed multiple times for each part of the model since the software does not support the editing and correction of the texture map after its production. Therefore, several tests are necessary to select the best images for texture rendering each part of the model. Moreover, in a number of cases, colour calibration had to be applied prior to the texture rendering, in order to correct discrepancies which occurred during photography, regarding brightness and white balance. A typical example was the dome of the church, which was poorly lit, due to the difficulty of tuning the studio flash and camera, and subsequently differed significantly from the other images in terms of brightness (Figure 7).

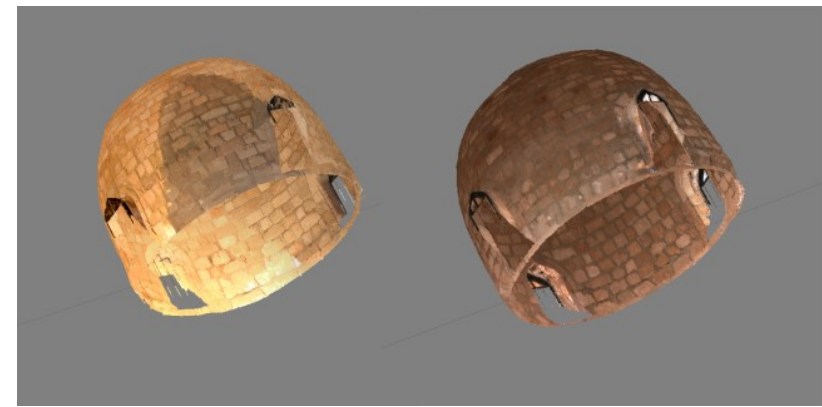

Figure 6. The dome of the church before (left) and after (right) colour calibration.

As previously mentioned, even after the necessary tests took place, the texture map for each of the parts of the model of the church of St Spyridon presented problems regarding radiometry. These problems were confronted with the use of Adobe Photoshop CC software. Another problem of the texture map was the way it was displayed on the image. More specifically, the form of "collage" of each texture map instead of a development makes it difficult to identify the position of each area on the model, especially when the texture is divided into very small pieces, thus making the editing of solely the texture map image virtually impossible (Figure 8).

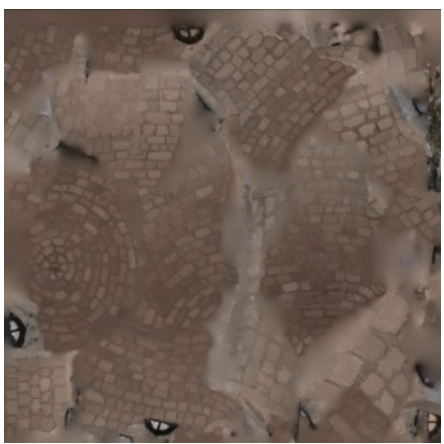

Figure 7. Texture map of the dome.

The map format could be improved with the use of additional software which would rebuild the texture map. This has been attempted with the use of Pixologic's ZBrush, which rebuilds the surface of the model by simplifying and reducing the triangles which represent the surface and allows the user to choose how the texture map will be displayed on the image, either by selecting among some defaults or by manually pointing how the map should be divided. This has been practiced in the case of the dome, which could easily be presented in the form of a development, due to its symmetric layout. However, this method was not applied in other parts of 
the model, mainly due to the lack of time, as well as the difficulty in defining the development of the texture map, since none of these parts presented the same symmetry and homogeneity as the dome. It should also be noted that the oversimplification of the surface would remove important details from the surface of the model and therefore, the method of reconstructing the texture map anew was not preferred.

However, the Adobe Photoshop CC software provides real-time view and editing of the textured model. It is possible to edit the texture map image while simultaneously viewing the results on the textured model (Figure 9). Thus, radiometric changes where applied on the texture maps, concerning the brightness and shadows. In many cases, it was necessary to correct the brightness at the edges of each of the individual models in which the church was divided, so that no abrupt colour changes were made. As for the exterior of the church, the necessary corrections regarded the color of the roof tiles, as the photography had taken place during different times of the day, resulting in a difference of the lighting. It was also required to remove the shadows created by the adjacent building and the elements of the church itself.
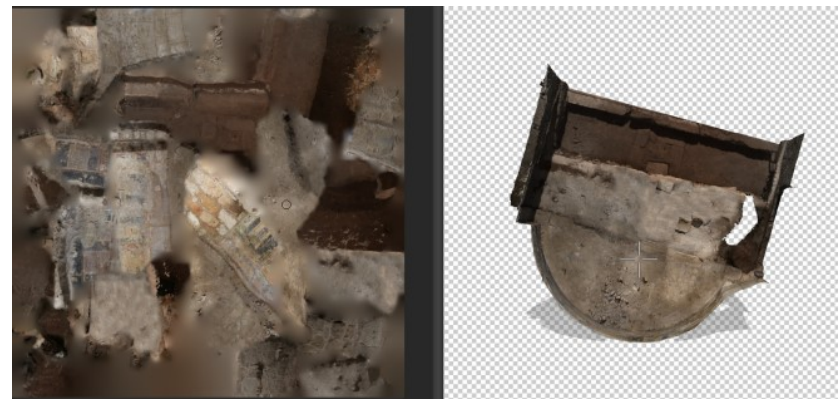

Figure 8. Real-time editing of the textured model in Adobe Photoshop CC.

The tools that were used to adjust the brightness and contrast throughout each texture map were Brightness/Contrast, Exposure and Vibrance. In cases were smaller parts of the texture maps had to be edited, the tools Dodge and Burn were used, to increase and reduce brightness, respectively. In addition, the colour correction, of the roof tiles (Figure 10), was achieved with the use of the Selective colour tool, which adjusts the percentage of each colour in each channel of the image.

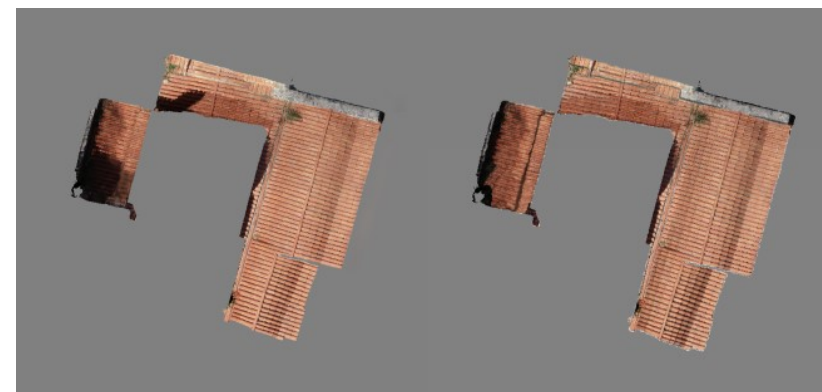

Figure 9. The roof tiles before and after colour adjustments.

\subsection{Stereoscopic (3D) Video}

For the presentation and promotion of the church of St Spyridon using the three-dimensional photorealistic model a stereoscopic video was created, with the use of Autodesk's 3DS Max software.
Initially, it was decided that two routes would be created, one for the exterior and one for the interior of the church. After all parts of the textured model were inserted in the software environment, two cameras were created for each route and the number of frames was set in order to define the length of each video, as well as the speed of the camera. Thus, the first camera covering the exterior of the church was set to 1000 frames, corresponding to 33 seconds of video, while the second camera capturing the interior of the church was set to 1500 frames, which correspond to about 50 seconds of video. In both cases, the video speed is set to $30 \mathrm{fps}$.

Moreover, the cameras were set to the targeted type, so that the camera would point at the center of the object. For this reason, two paths had to be designed for each camera, the first being the path the camera would follow and the second defining the direction and orientation of the camera's optical axis.

Finally, the lens for each camera was determined taking into consideration how large an area of the object would be displayed in each frame of the video. After a series of tests and based on the criterion of the best presentation of the interior of the church, the $8 \mathrm{~mm}$ lens was used for both cameras, with a field of view of $100^{\circ}$. For a smoother transition from one camera to the other, the last nodes of the paths of the first camera were the first ones for the paths of the second camera.

Finally, the rendering process was performed for each camera, creating the first (non-stereoscopic) video, after appropriately lighting the object, defining its surroundings and activation the respective animations. One of these animations was the movement of the door at the entrance of the church, opening it upon the entrance of the camera. Key-movements were created and placed in the right frames of the video to synchronize the movement of the door with the movement of the camera.

The video's resolution was set to $1920 \mathrm{x} 1080 \mathrm{px}$ and it was exported in avi format without compression, so that the quality and definition of the model would not be degraded.

Consequently, the stereoscopic video was created using the cameras of the previous video. All the parameters mentioned above, related to the number of frames, camera lens etc, remained the same. Two new auxiliary cameras were added for each of the already existing cameras, in order to facilitate the stereoscopic perception of the new video. Out of these cameras, the one on the left represents the left eye vision and the one of the right represents the right eye vision. The rendering process was the same as before, with the exception that it was performed separately for each new camera. In order to combine the two videos that emerged for each camera into a single stereoscopic view, Adobe After Effects $C C$ software was used, selecting the Blue Red LR method. The final stereoscopic video of the church of St Spyridon was a minute and 24 seconds long and should be watched with the use of "anaglyph" glasses (BR) for optimal view (Figure 11). 


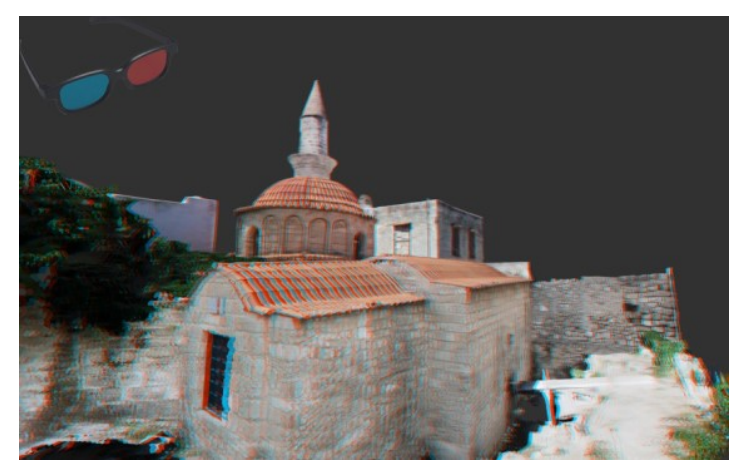

Figure 10. Still of the stereoscopic video which should best be watched with "anaglyph" glasses.

\subsection{DHOP platform}

Finally, an interactive tour of the three-dimensional photorealistic model of the church of St Spyridon was created, using the $3 D H O P$ (3D Heritage Online Presenter) platform (http://3dhop.net/).

$3 D H O P$ is an open source platform for the creation of interactive high-resolution presentations of three-dimensional models oriented to the field of cultural heritage. The platform enables three-dimensional models to be displayed on a single web page, modifying HTML code with additional HTML and JavaScript elements. For this application, it was decided that the files would load locally, bypassing the server and without the need for a web server installation. To this end, the browser would be forced to accept the local files, by adding the "allowfile-access-from-files" parameter to the Google Chrome browser shortcut destination.

The 3DHOP platform can handle three different types of geometries: small-sized three-dimensional models (less than 1 MB), large three-dimensional models (up to 100 million triangles) and point clouds, also up to 100 million points. In this application, the three-dimensional textured model was used, which had to be properly prepared and modified in order to be added to the platform (Figure 12).

To this end, all models were converted from .obj to .ply and then to Nexus (.nxs). Nexus is a collection of high-resolution three-dimensional modeling tools in OpenGL $(\mathrm{C}++$ and JavaScript), storing the texture of the model internally. The .nxs files can then be compressed into the .nxz format for easier management, however the uncompressed .nxs format performs better in local use tasks, as is the case for this application. (Ponchio, 2019) Moreover, the georeferencing of the models remains stored in them and since $3 D H O P$ uses a coordinate system in which the $\mathrm{X}, \mathrm{Y}$ and $\mathrm{Z}$ axes are identical to those of the model's coordinate system, no additional changes in position, rotation and scale were required. (3DHOP Development Team, 2020)

For the creation of the interactive tour on the $3 D H O P$ platform, parts of the code of other applications provided as examples/tutorials on the 3DHOP website were used. However, modifications to the HTML code were required, with the addition of JavaScript elements.

Initially, the parts of the model were grouped depending on their location and a toolbar was created, containing the following:

- Home, to return to the platform's home screen
- Zoom in and Zoom out options to navigate closer or further from the model

- $\quad$ Lighting and Light Control options that allow the use of additional lighting and adjustments to its position

- Hotspots to activate the areas of interest of the church

- $\quad$ Measure, for measuring the model (in m)

- The Pick point tool, to display the local coordinates of any point of the church

- Full screen for full screen navigation

- The Help option to display instructions on the controls used for browsing.

In regard to the Hotspots tool, seven bullets were placed at various points of interest of the church. Clicking on each of them leads to display of a table containing an image depicting the particular section of the church, as well as information about the history and architecture of the church. For this purpose, an additional JavaScript file was created, which contained the functions necessary to associate each hotspot with the display of the corresponding table. Using the same JavaScript file, the Help option was also associated with the appearance of the navigation panel.

Also, another menu was created, allowing the display of only certain parts of the church. In particular, it is possible to conceal the exterior parts of the church so that only the interior ones are visible, and for the interior, the option to only show the ceiling or floor is available. In addition, there is an option to solely display the crypt.

The Sphere Trackball tool was used for browsing the model, allowing complete and free rotation and movement across all axes. The only two parameters that had to be specified were the original distance of the trackball from the object and the minimum and maximum distance of view. Its position can be altered by double-clicking anywhere on the model, while movement is possible by pressing the mouse washer.

Finally, and HTML file was created for interactive browsing on the $3 D H O P$ platform, accompanied by eight JavaScript files and two .css files.

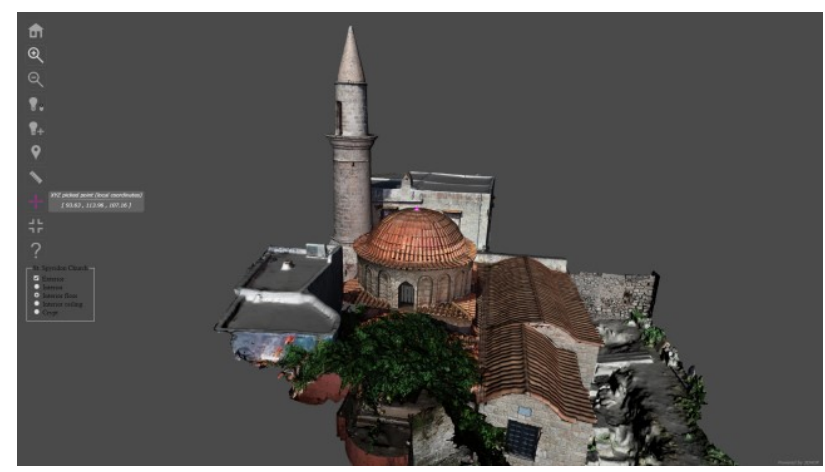

Figure 11. Screenshot of the "Measure" tab in the 3DHOP app.

\section{CONCLUSIONS}

First, it is worth mentioning the great contribution of TheoLt software used for the collection of the geodetic data, mainly regarding the ability for real-time control of each measured point and section during field work. Therefore, with each measurement of the section lines and the targets placed inside and outside the monument it was possible to achieve a better 
understanding of the general image of the church, as well as directly control each measured point, thus avoiding blatant errors. Moreover, its main advantage was that it eliminated the geodetic data processing, decisively reducing the required processing time. Additionally, the importance of collecting the required volume of data was made clear during field work. Collecting less information could lead to shortages in the final drawings and three-dimensional model, while too much could lead to implications and problems during the editing process, while at the same time significantly increase processing time.

Having completed the work cycle and after evaluating its results, one must consider the mistakes that have been made and how they could have been prevented. In this geometric documentation, the most profound problem regarded the roof tiles and the dome of the church. After acquiring the necessary knowledge and experience, it is concluded that more attention should have been paid to those specific sections of the monument, with more scanning positions in order to acquire more details, UAV photographs taken from positions closer to the object, as well as photographs of the roof using the DSLR camera.

Moreover, the video production was a relatively unknown process, as $3 D S$ Max is a fairly complex and specialized software mainly focused on creating games and virtual reality applications. A number of attempts were required mainly regarding the routes of the camera, the speed and lens and it took a different, directorial approach of the church to crate the best possible result for the viewers.

Additionally, concerning the creation of the interactive tour, $3 D H O P$ is a platform with a variety of features, only a few of which were used as indicators of the platform's possibilities in this application. The final result can certainly be improved and enriched, but this requires very good knowledge of the platform and coding, in general. This is a time consuming and fairly demanding process, which has not been followed in this instance, however addition features could be added to the tour, such as the creation of a new hotspot for the stereoscopic video, the presentation of all the measured sections and the corresponding drawings and orthophotographs, as well as the improvement of the lighting effects and surroundings of the model.

The enriched version of the platform with the aforementioned elements, as well as the drawings themselves, can be used as a means of promotion of the church by the Ephorate of Antiquities of Dodecanese, for both educational and professional purposes (use of the drawings by other engineers, architects and archaeologists).

Finally, through this work, the importance of cooperation has emerged, both among individuals of the same specialty and among different specialties, such as surveying engineers and archaeologists, as each of them has to offer valuable know-how in order that the final result of the geometric documentation may be as complete and accurate as possible.

\section{ACKNOWLEDGEMENTS}

We would like to thank the Ephorate of Byzantine Antiquities of Dodecanese for granting permission to work on the site and for their hospitality while working on the project in Rhodes, as well as for the contribution regarding all historical and architectural information needed. Moreover, we would like to thank Ms A.-M. Boutsi for her valuable advice and help regarding the setup of the interactive tour of the church of St. Spyridon, using the $3 D H O P$ platform.

\section{REFERENCES}

3DHOP Development Team, 2020. 3DHOP: 3D Heritage Online Presenter, http://3dhop.net/.

3DHOP Development Team, 2020. 3D models preparation, http://3dhop.net/documentation/3DHOP_Models_Preparation.p df.

Agisoft Metashape Development Team, 2019. Agisoft Metashape User Manual: Professional Edition, Version 1.5, 2931 .

Blake B., Grundon I., 2008. Training for TheoLt Field Survey.

Hadjidemetriou G., 2015. Survey Guide (ArchDoc \& IPW3 edition), Raymond Lemaire International Center of Conservation \& Technologie campus Gent, 13-33.

Ponchio F., Visual Computing Lab, 2019. Nexus: adaptive 3D (C++ and JavaScript), http://vcg.isti.cnr.it/nexus/.

Volanakis I., 2000. The church of St. Spyridon in the medieval city of Rhodes. Minutes of International Scientific Conference: Rhodes: 2400 years, the city of Rhodes from its creation to its occupation by the Turks (1523), Athens, Greece, 367-374.

Westoby et al., 2012. Structure-from-motion Photogrammetry: A low-cost, effective tool for geoscience applications. 